\title{
An Observational Study of Personal Ultraviolet Dosimetry and Acute Diffuse Reflectance Skin Changes at Extreme Altitude
}

\author{
Ivy Cheng, MD, FRCP; Alex Kiss, PhD; Lothar Lilge, $\mathrm{PhD}$ \\ From the Emergency Department, Sunnybrook Health Sciences Center, North York, Ontario, Canada (Dr Cheng); Institute of Clinical Evaluative \\ Sciences at Sunnybrook Health Sciences Center, North York, Ontario, Canada (Dr Kiss); and the University of Toronto, Toronto, Ontario, Canada \\ (Dr Lilge).
}

\begin{abstract}
Objective.-To determine the level of UV radiation at extreme altitude and to assess the effect it has on the skin.

Methods.-Fifteen expeditioners and 10 Sherpas were assessed during a climbing expedition on the north side of Mt Everest $(8848 \mathrm{~m})$. UV exposure measurement and diffuse skin reflectance spectrophotometry were performed at the beginning and end of the expedition.

Results.-Over the course of the expedition, the expeditioners and Sherpas received a median dose of 93.6 (interquartile range [IQR], 61.0-102.8) and 102.5 (IQR, 72.2-117.8) minimal erythemal doses (MEDs) of UV radiation. The maximum dosage exceeded $106 \pm 1.4$ MEDs. Using reflectance spectrophotometry, expeditioner and Sherpa melanin-hemoglobin increased by $83.6 \%$ (IQR, -1.5 to $89.8 \%$ ) and $24.7 \%$ (IQR, 22.4 to $61.5 \%$ ) for exposed skin, respectively. The amount of subcutaneous lipid-water decreased by a factor of 196.6 (IQR, 52.1-308.4) and 46.7 (IQR, 1.8-1156.5), for expeditioners and Sherpas, respectively.

Conclusions.-This expedition's participants received massive doses of UV radiation during their time at high altitude. In many individuals this was similar to the annual exposure of northern European office-workers (100 MEDs). Diffuse skin reflectance spectroscopy revealed considerable subcutaneous lipid loss, skin dehydration, and increased melanin in keeping with these levels of exposure.
\end{abstract}

Key words: ultraviolet exposure, skin, extreme altitude, diffuse reflectance, spectrophotometry, attenuation, mountain medicine

\section{Introduction}

Ultraviolet (UV) radiation is associated with a wide range of medical problems. ${ }^{1}$ Cyclists, ${ }^{2}$ triathletes, ${ }^{3}$ polar explorers, ${ }^{4}$ and high altitude mountaineers ${ }^{5}$ have all been shown to receive considerable amounts of UV light exposure. Since the advent of reliable, portable equipment, studying UV dosage and acute skin changes in nonlaboratory settings has never been easier. Therefore, we set out to measure the dose of UV radiation received by members of an expedition to Mt Everest and to

Disclosure statement: The Ontario Centers of Excellence funded this study. Drs. Cheng, Kiss, and Lilge have no conflicts of interest.

Trial registration number: NCT00685438 ClinicalTrials.gov

Corresponding author: Ivy Cheng, MD, FRCP, Emergency Department, Sunnybrook Health Sciences Center, C753-2075 Bayview Avenue, North York, Ontario, Canada, M4N 3M5 (e-mail: ivy. cheng@sunnybrook.ca). quantify the impact this had on their skin by using diffuse reflectance spectrophotometry.

\section{Methods}

This prospective, observational study was undertaken on the north side of Mt Everest $(8848 \mathrm{~m})$ from April 25 to June 4, 2007. The Sunnybrook Health Sciences Centre (Toronto, Canada) research ethics board approved the study. Twenty-five individuals (10 Sherpas, 15 expeditioners) from a single expedition consented to participate in the study. Participants were classified into 4 groups: those only undertaking the climb of Lhakpa Ri (7045 m, May 9) [L]), those making the first summit attempt on Everest (Summit Attempt, May 22 [E1]), those making the second attempt on Everest (Summit Attempt, June 4 [E2]), and Sherpa (Lhakpa Ri, both Everest Summit Attempts [S]). 
Personal UV dosimetry was measured with the Viospore (BioSense, Siegburg, Bornheim, Germany) monitor. This device uses a film of immobilized Bacillus subtilis spores (Figure 1A). Its inactivation is known to correlate well with UV-B exposure in a minimal erythema dose (MED) range of 290 to $380 \mathrm{~nm}$ for Caucasian skin (ie, sunburn). ${ }^{6}$ The dosimeter functions independent of humidity, and temperature $\left(-20^{\circ}\right.$ to $+70^{\circ} \mathrm{C}$ ) and has been used successfully up to an altitude of $7100 \mathrm{~m}^{7}$ Participants wore the dosimeters continuously for the duration of the expedition. Two devices were sited - the first was located at the crown of the head (Figure 1B), and a second device was placed in the axilla to act as a control. On ascending above base camp (BC), the dosimeter was replaced in all 4 groups.
At the end of the expedition, dosimeters were sent to BioSense for MED calculation. Total UV dose was determined by adding dosimeter readings at $\mathrm{BC}$ and above BC. Daily UV dose was calculated by dividing the total dose with the number of days wearing the dosimeter.

To determine acute skin changes for unexposed (chest) and exposed skin (cheek), diffuse reflectance spectrophotometry was performed before and after the expedition. An S2000 Ocean Optics spectrophotometer (Dunedin, FL) connected to a laptop-powered white LED (SSL-LX3054UWC/B; Lumex, Palatine, IL) was used. Because none of the expedition laptops worked at elevations above BC $(6475 \mathrm{~m})$, reflectance measurements were limited to elevations at or below $6475 \mathrm{~m}$ and
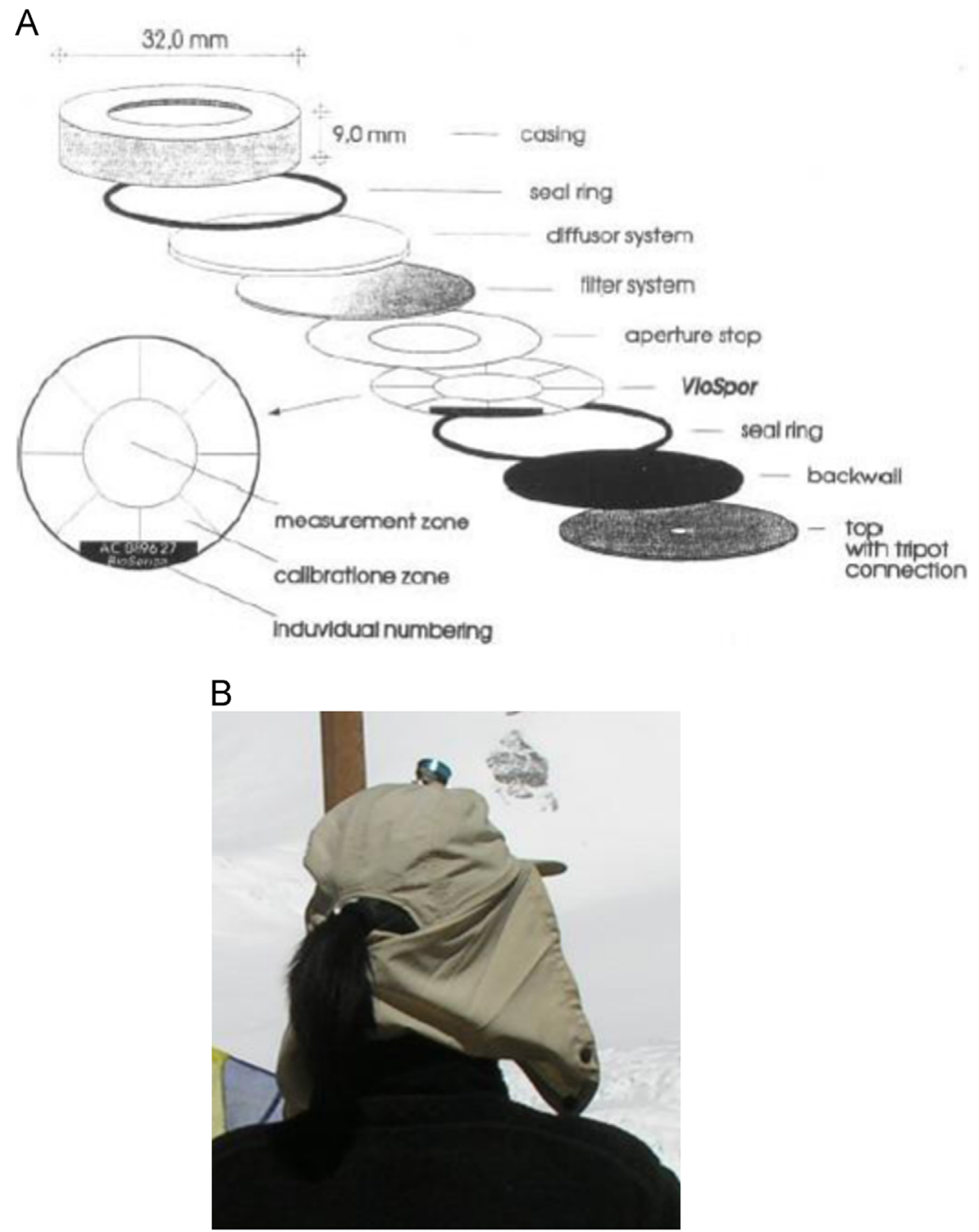

Figure 1. (A) Viospore monitor. ${ }^{7}$ This 15 -g device uses a film of Bacillus subtilis spores. (B) Viospore monitor on crown of head. 
Table 1. Study population and ascent profile

\begin{tabular}{|c|c|c|c|c|c|c|c|c|c|}
\hline \multirow[b]{2}{*}{ Group } & \multirow[b]{2}{*}{ No } & \multirow[b]{2}{*}{ Age (years) } & \multirow[b]{2}{*}{ Male } & \multicolumn{3}{|c|}{ No. reaching elevation } & \multicolumn{3}{|c|}{ Days $($ mean $\pm S D)$} \\
\hline & & & & $6500 \mathrm{~m}$ & $7045 \mathrm{~m}$ & $\geq 8000 \mathrm{~m}$ & $\overline{\text { Below BC }}$ & Above $B C$ & Total \\
\hline Expeditioners & 15 & $27-62$ & 11 & 15 & 11 & 9 & & & \\
\hline Lhakpa Ri & 4 & & 3 & 4 & 2 & 0 & 7 & 9 & 16 \\
\hline Everest $1^{a}$ & 6 & & 4 & 4 & 4 & 5 & $14.3 \pm 5.4$ & $19.3 \pm 5.1$ & $33.7 \pm 1.6$ \\
\hline Everest $2^{a}$ & 5 & & 4 & 4 & 4 & 4 & $16.8 \pm 1.6$ & $26.4 \pm 2.3$ & $43.2 \pm 1.3$ \\
\hline Sherpa & 10 & $21-46$ & 10 & 10 & 10 & 9 & $6.7 \pm 2.8$ & $29.9 \pm 5.5$ & $36.6 \pm 5$ \\
\hline
\end{tabular}

BC, base camp (elevation, $6500 \mathrm{~m}$ ).

${ }^{a}$ Includes support staff (1 per group) with no ascent above BC.

at temperatures between $-5^{\circ}$ and $20^{\circ} \mathrm{C}$. Although LED intensity was affected by temperature ( $10 \%$ higher emissions at $4^{\circ} \mathrm{C}$ compared with $20^{\circ} \mathrm{C}$ ), spectral emission characteristics were not altered. This was confirmed in a cold laboratory after return from the expedition.

The spectrophotometry system uses white light to illuminate the skin, and superficial layers attenuate light by absorption and scattering. Reflected light is recorded and subsequently converted to attenuation spectra where specific wavelengths represent different skin components. Oxyhemoglobin-melanin (510-600 nm), erythema (double peak at 540 and $575 \mathrm{~nm}$ ), and lipids-hydration (770-780 nm) were compared before and after the expedition.

Total UV dosimetry was calculated and compared with recommended industry standards for UV exposure (International Commission of Illumination $[\mathrm{CIE}]^{8}$ ). Ultraviolet exposure comparison between the Sherpas and expeditioners (Wilcoxon rank sum) was calculated. Total and daily UV dosages between base camp and above were performed by paired Student's $t$ test once normalcy (Shapiro-Wilks) was determined. Comparison of skin attenuation between Sherpas and expeditioners in the 510 - to $600-\mathrm{nm}$ and $770-$ to $780-\mathrm{nm}$ range was accomplished by the Wilcoxon rank sum test.

All analyses were carried out using SAS version 9.1 (SAS Institute, Cary, NC). Descriptive statistics (median, interquartile range) were calculated using SPSS version 21.0 (IBM, Armonk, NY).

\section{Results}

Table 1 and Figure 2 provide the participants' demographics, elevation profile, dosimeter distribution, and subsequent grouping. After the expedition, $72 \%$ of the dosimeters and $96 \%$ of the participants' skin reflectance spectra were analyzed. Fourteen of the dosimeters were lost during the expedition and therefore are unavailable for analysis.

At altitudes higher than $6500 \mathrm{~m}$, the temperature occasionally decreased to less than $-20^{\circ} \mathrm{C}$, and therefore we had the potential to underestimate the UV dose

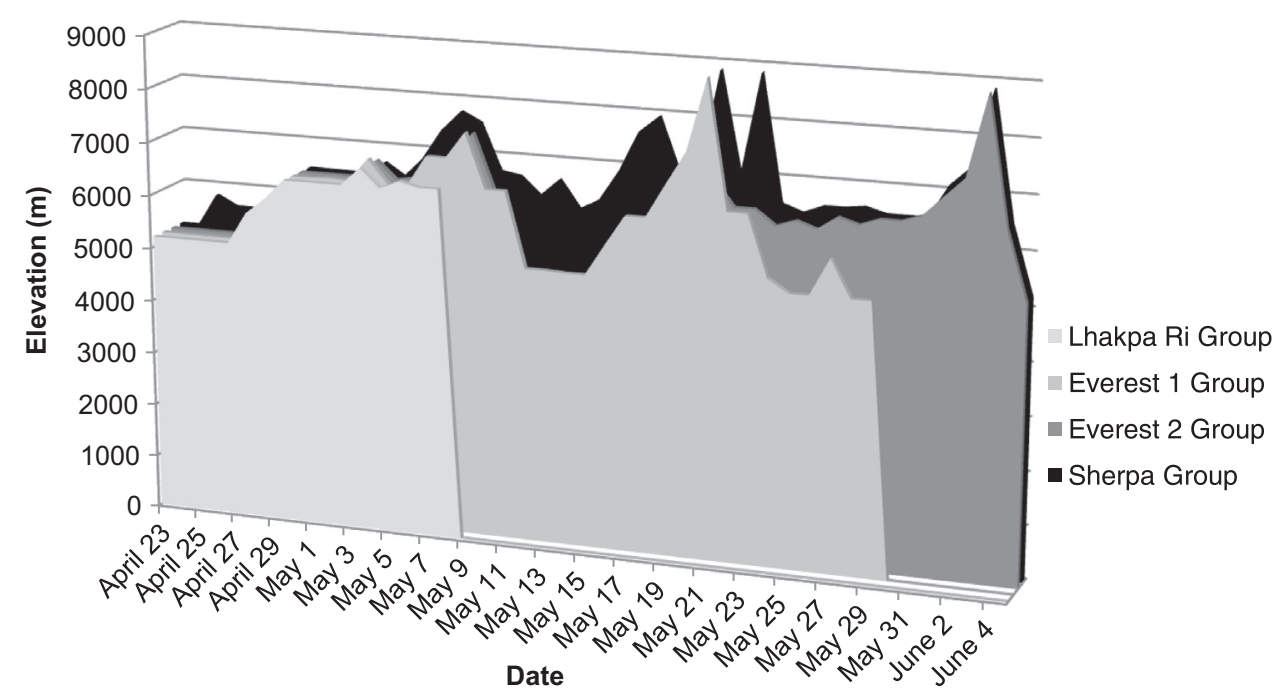

Figure 2. Duration and elevation profiles of the 2007 north Everest expedition. 
recorded by the dosimeter. Results are summarized in Table 2. During the study period, expeditioners and Sherpas were exposed to a 6.7 to 13.0 times the CIE recommended daily threshold limit values (TLV) of 0.43 MED UV radiation per 8-hour workshift. ${ }^{5}$ In 44 days, the E2 and Sherpa groups exceeded the estimated annual exposure of a northern Europe indoor worker of 100 MEDs. This was emphasized by the discovery that 2 dosimeters were exposed beyond their calibrated dynamic range of 90 MEDs. At BC, the expeditioners were exposed to a significantly lower daily UV dose than the Sherpas $(P=.01$, Wilcoxon rank sum).

Above BC, the expeditioners, Sherpas, and combined (Sherpa and expeditioner) were exposed to significantly higher median differences in cumulative UV dosage of +58.5 (IQR, 32.6-77.2), +52.2 (IQR, 23.6-57.0), and +52.2 (IQR, 25.8-63.5) MEDs, respectively, than at BC (paired t test, Shapiro-Wilks). The Sherpas and combined groups were exposed to +2.7 (IQR, 2.1-4.5) and +1.3 (IQR, 0-2.6) significantly greater daily MEDs at $\mathrm{BC}$ compared with above BC, respectively (paired $t$ test, Shapiro-Wilks).

Skin changes are summarized in Table 3. Skin melanin-hemoglobin content for all groups increased (ie, the skin darkened) after the expedition. In expeditioners, unexposed melanin-hemoglobin skin content also increased; however, a decrease (ie, lightening of the skin) occurred in the Sherpas. Erythema, a double peak at $540 \mathrm{~nm}$ and $575 \mathrm{~nm}$, was not detected (Figure 3). ${ }^{9}$ The skin lipid-water content after expedition fell by almost a factor of 200 compared with preexpedition measurement (Table 3, Figure 3). The 760-nm lipid peak ${ }^{10}$ was lost in almost all participants, signifying subcutaneous fat loss (Figure 3A). One exception was a Sherpa who did not climb any higher than $7700 \mathrm{~m}$ and did not lose his lipid peak (Figure 3B). Another was a multisummit Sherpa whose preexpedition spectrum did not have a lipid peak and had higher attenuation levels than other participants' postexpedition spectra (Figure 3C). Comparing melanin-hemoglobin and lipidwater content identified no significant differences between the Sherpas and expeditioners.

\section{Discussion}

We found that the massive UV exposure sustained during an expedition to Everest is greater than 100 times the minimal dose required to cause a sunburn for Caucasian skin. The dosages surpassed the recommended occupational health standards and in some cases exceeded the cumulative annual exposure of an indoor office worker. Despite evidence that skin darkened and

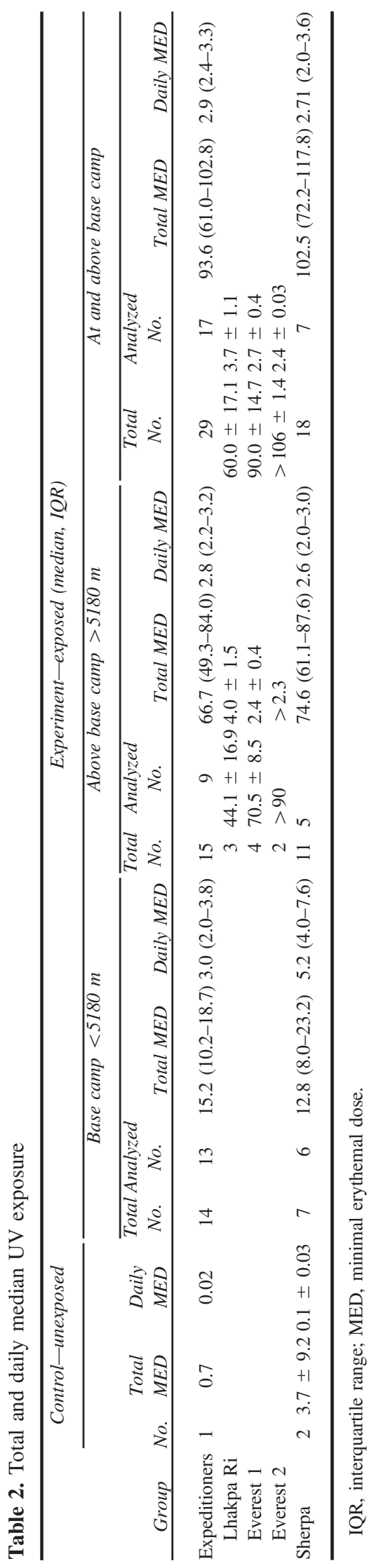


lost subcutaneous fat and water, sunburn was not detected.

The patterns of mean daily UV exposure among expeditioners and Sherpas can be explained in part by differences in behavior. Sherpa exposure was greater at $\mathrm{BC}$ because they worked outdoors for most of the day. Above BC, Sherpa exposure was lower. This may be explained by the preference of Sherpas to climb at night and, importantly, much quicker than expedition members who would have been exposed for longer periods during the middle of the day.

The absence of skin erythema, the hallmark of acute UV-related skin injury, may have been related to using appropriate UV barriers, such as clothing and brimmed hats, seeking shelter, or recovering before measurement took place. Although skin darkening was most likely to be caused by UV exposure, subcutaneous fat and water loss may have also been the result of dehydration and caloric loss associated with climbing at high altitude. The combination of UV radiation, hypoxia, high-altitude exertion, and malnutrition is significant as immunosuppressive risk ${ }^{1,11-13}$ and carcinogenesis ${ }^{1}$ are compounded.

Consequently, extreme UV exposure in the climbers has important health implications because of its risk of skin cancer, cataract formation, and acute immunosuppression. These adverse health outcomes have potential long-term effects with a significant global burden of disease. ${ }^{1}$ Because Nepal is a low-resource country, the lack of sun protection and treatment programs may further exacerbate Sherpa risk compared with the expeditioner. During any high altitude activity, it is essential to mitigate the health risks of UV radiation by inexpensive and simple strategies: limit sun exposure as much as possible by seeking shelter or shade, wear adequate UV-protected clothes and sunglasses, and continually apply and reapply high-factor sunblock creams. Adequate hydration and nutrition are important to minimize dehydration and fat loss.

\section{Limitations}

There were several limitations to this study. The investigator could not continually monitor proper dosimeter placement, resulting in potential inaccuracy and underestimation of dose. Control monitors could have been accidentally exposed. Alternatively, the controls could have been exposed from inadequate clothing protection, ${ }^{14}$ thereby underestimating UV doses. There was heavy reliance on the instruments' robustness, accuracy, and prior biophysics literature.

Erythema may have been missed because only before and after expedition spectroscopic readings were performed. Unwashed skin or barrier creams could 

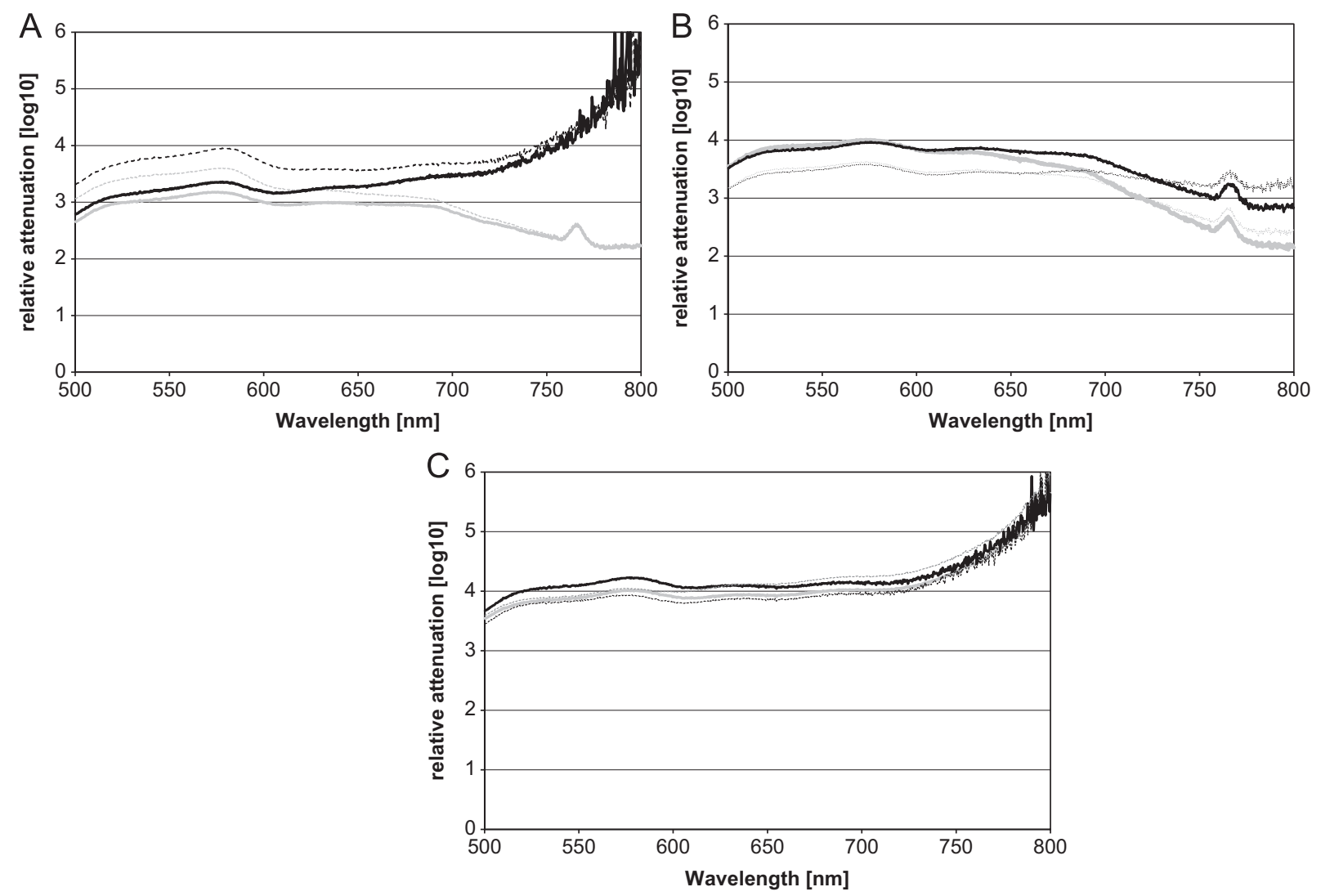

Figure 3. Three samples of spectrophotometer spectra: (A) Everest 2, summitter. (B) Sherpa, nonsummitter. (C) Sherpa, Everest multisummitter. Preexpedition, April 23 to May 1 [all groups]; postexpedition, May 9 [group L] to June 4 [group E2, S]; preexpedition spectra of nonexposed skin (chest)—dotted gray; preexpedition spectra of exposed skin (cheek)—solid gray; postexpedition spectra of nonexposed skin (chest)—dotted black; postexpedition spectra of exposed skin (cheek)—solid black.

hypothetically skew readings; however, modern sun creams lose their optical properties once absorbed, with no impact on spectroscopic readings. Extracting skin chromophore concentration by using chromophore indices would have been of higher scientific value. However, our diffuse reflectance spectrophotometer was a shortsource detector-designed to detect epidermal and upper dermal changes only. Chromophore algorithms do not work for short-source detector distances. Because of this design, algorithms using chromophore indices were not applicable.

\section{Conclusions}

The participants in this expedition were exposed to significant amounts of UV radiation. In contrast to the obvious life-threatening physical hazards of mountaineering, UV radiation is subtle with no immediate mortality. However, it should not be underestimated. Further study is required to determine whether extreme altitude climbers are at greater risk for UV-related morbidity or mortality.

\section{Acknowledgments}

The authors are grateful to Dr Hans Holtschmidt (Biosense Laboratory for Biosensory Systems, Bornheim, Germany) for Viospore monitors and analysis, to Gamble Technologies (Ottawa, Ontario) for spectrophotometer, to DCXP (Adelaide, Australia)/Arun Trekking (Kathmandu, Nepal) participants, and to Dr Jeremy Windsor for supporting this study in part.

\section{References}

1. Lucas R, McMichael T, Smith W, Armstrong B. Solar Ultraviolet Radiation: Global Burden of Disease From Solar Ultraviolet Radiation. Geneva, Switzerland: World Health Organization; Environmental Burden of Disease Series, No 13; 2006.

2. Moehrle M, Heinrich L, Schmid A, Garbe C. Extreme UV exposure of professional cyclists. Dermatology. 2000;201: 44-45.

3. Moehrle M. Ultraviolet exposure in the Ironman triathlon. Med Sci Sports Exerc. 2001;33:1385-1386. 
4. Gies P, Watzl R, Javorniczky J, et al. Measurement of the UVR exposures of expeditioners on Antarctic resupply voyages. Photochem Photobiol. 2009;85:1485-1490.

5. Moehrle M, Dennenmoser B, Garbe C. Continuous longterm monitoring of UV radiation in professional mountain guides reveals extremely high exposure. Int $J$ Cancer. 2003;103:775-778.

6. Quintern LE, Furusawa Y, Fukutsu K, Holtschmidt H. Characterization and application of UV detector spore films: the sensitivity curve of a new detector system provides good similarity to the action spectrum for UV-induced erythema in human skin. J Photochem Photobiol B. 1997;37:158-166.

7. Moehrle M, Korn M, Garbe C. Bacillus subtilis spore film dosimeters in personal dosimetry for occupational solar ultraviolet exposure. Int Arch Occup Environ Health. 2000;73:575-580.

8. Ultraviolet Radiation. International Programme on Chemical Safety. Environmental Health Criteria 160. Geneva, Sqitzerland: World Health Organization; 1994.
9. Andersen PH, Bjerring P. Spectral reflectance of human skin in vivo. Photodermatol Photoimmunol Photomed. 1990;7:5-12.

10. Spinelli L, Torricelli A, Pifferi A, Taroni P, Danesini GM, Cubeddu R. Bulk optical properties and tissue components in the female breast from multiwavelength time-resolved optical mammography. J Biomed Opt. 2004;9:1137-1142.

11. Hug DH, Hunter JK, Dunkerson DD. Malnutrition, urocanic acid, and sun may interact to suppress immunity in sojourners to high altitude. Aviat Space Environ Med. 2001;72:136-145.

12. Bailey DM, Castell LM, Newsholme EA, Davies B. Continuous and intermittent exposure to the hypoxia of altitude: implications for glutamine metabolism and exercise performance. Br J Sports Med. 2000;34:210-212.

13. Nieman DC. Current perspective on exercise immunology. Curr Sports Med Rep. 2003;25:239-242.

14. Davis S, Capjack L, Kerr N, Fedosejevs R. Clothing as protection from ultraviolet radiation: which fabric is most effective? Int J Dermatol. 1997;36:374-379. 